\title{
The nonlinear photon transfer curve of CCDs and its effects on photometry
}

\author{
Bin $\mathrm{Ma}^{1}$, Zhaohui Shang ${ }^{2,1}$, Lifan Wang ${ }^{3,4}$, Yi Hu${ }^{1}$, Qiang $\mathrm{Liu}^{1}$, Peng $\mathrm{Wei}^{1}$ \\ ${ }^{1}$ National Astronomical Observatories, Chinese Academy of Sciences, Beijing, China; \\ ${ }^{2}$ Tianjin Normal University, Tianjin, China; \\ ${ }^{3}$ Purple Mountain Observatory, Chinese Academy of Sciences, Nanjing, China; \\ 4Texas A\&M University, College Station, Texas, USA;
}

\begin{abstract}
The photon transfer curve (PTC, variance vs. signal level) is a commonly used and effective tool in characterizing CCD performance. It is theoretically linear in the range where photon shot noise dominates, and its slope is utilized to derive the gain of the CCD. However, recent researches on different CCDs have revealed that the variance progressively drops at high signal levels, while the linearity shown by signal versus exposure time is still excellent and unaffected. On the other hand, bright stars are found to exhibit fatter point spread function (PSF). Both nonlinear PTC and the brighter-fatter effect are regarded as the result of spreading of charges between pixels, an interaction progress increasing with signal level. In this work we investigate the nonlinear PTC based on the images with a STA1600FT CCD camera, whose PTC starts to become nonlinear at about $1 / 3$ full well. To explain the phenomenon, we present a model to characterize the charge-sharing PSF. This signal-dependent PSF can be derived from flat-field frames, and allow us to quantify the effects on photometry and measured shape of stars. This effect is essentially critical for projects requiring accurate photometry and shape parameters.
\end{abstract}

Keywords: CCD, Photon Transfer Curve, Nonlinearity, Point Spread Function, Photometry Precision

\section{INTRODUCTION}

The photon transfer curve (PTC, variance vs. signal level ${ }^{1}$ is a fundamental tool in characterizing ChargeCoupled Device (CCD) performance. Its primary purpose is to determine the "gain" parameter of a CCD system which denotes the conversion from the number of collected electrons to the digital number read out by the amplifier. Using the gain, many of the other performance parameters such as readout noise, dark current, full well etc. are converted in physical unit of $e^{-}$.

The variance of pixel values in a flat frame with uniform illumination consists of:

i) readout noise, which is constant and usually small in modern CCDs;

ii) photon shot noise, which follows Poisson noise and is proportional to the signal level;

iii) actual signal fluctuation between pixels, which is caused by the Quantum Efficiency (QE) difference and is proportional to the square of the signal level.

If a pair of flat frames are taken with identical illumination and exposure parameters, their difference will remove the QE-related sensitivity fluctuation. Therefore the common PTC is linear beyond extremely low signal, and its slope is the inverse of the "gain" parameter.

However, Ref.2 reported that the nonlinearity of PTC had been observed on CCDs from different manufacturers even though the signal increased excellently linearly with exposure time ( better than $1 \%$ ). In addition, the PTC nonlinearity progressively increased with signal level, and was in excess of $15 \%$ before saturation was reached. They carried out many tests and excluded some potential causes such as lateral diffusion of charge in

Send correspondence to Bin Ma: mabin22@gmail.com 
the undepleted region at the back of the imager, the clocking or transport of charge in the image area or serial register to the read out, the output amplifier, and the detector electronics. They concluded that the nonlinear PTC was related to the amount of charge collected within a pixel, and suggested that the mechanism behind nonlinearity was charge spreading or sharing according to spatial autocorrelation analysis. Their other work ${ }^{3}$ shows the nonlinearity is greater for thicker devices made from higher resistivity silicon.

To explain the phenomenon, they have presented a hypothesis ${ }^{4}$ that the collected charges in a pixel reduces the extend of the pixel's electric field, and consequently reduces the pixel's collection competitiveness with respect to its neighbors. Because the charge sharing increases linearly with signal, they expected the Point Spread Function (PSF) to broaden with signal, and indeed found that the PSF of laboratory spots rose linearly with their signal levels. This brighter-fatter effect was detected in scientific images as well $\frac{[5}{5}$ In addition, they suggested that the PTC could be well fitted to a quadratic function, the linear term of which should be a better estimator of gain.

Alternatively, Ref. [6 developed a Monte Carlo model to describe the process of signal dependent chargesharing. They formulated the probability of charge-sharing to be proportional to the instantaneous number of excess electrons in a pixel compared to the average number of electrons over all pixels. And the shared electrons could be received by either a random neighbor pixel or the neighbor with the lowest number of electrons. Then a Monte Carlo simulation, tracing individual electrons generated by Poisson-distributed photons, reconstructs the quadratic PTC with sub-Poisson noise. However, the model is a macro model, of which the sharing coefficient is empirical but not based on physical parameters.

In another model by Ref.7, the Coulomb forces from accumulated charges within all pixels of the CCD distort the drift electric field, making the boundaries of each pixel slightly dynamical and hence forthcoming charges probably drift on the boundaries. This model bridges both the lab tests and scientific observations: the displacement of the effective boundaries of a pixel at some signal level is derived from correlations in the lab flat frames, then it is applied in the scientific images and predicts the flux-dependent PSF with $\sim 20 \%$ accuracy. However, for a $n \times n$ region in question, there need $4 n^{2}$ coefficients for a pixel with four boundaries and $n^{2}$ neighbors, while there are only $n^{2}$ correlation measurements. Providing that each boundary is shared by two pixels, the measurements are still short by a fact of 2 . In order to solve the problem anyway, they have imposed ratios of coefficients addressing similar source-test separations.

We have found that the CCDs for the trio Antarctic Survey Telescopes (AST3) ${ }^{8}$ also exhibit signal dependent nonlinear PTC 9 The CCD cameras, STA1600FT, are designed and manufactured by Semiconductor Technology Associates, Inc.. They have 10, $560 \times 10,560$ pixels with a pixel size of $9 \mu \mathrm{m}$, corresponding to 1 arcsec on the focal plane of AST3. The CCDs work in frame transfer mode to avoid malfunction of mechanical shutter, and they have 16 amplifiers to speedup the readout. In this paper, we present an alternative model of charge sharing to study the nonlinear PTC. We will investigate the PTC and correlation in the lab flat frames in Sec. 2 In Sec. 3 we describe the model, which simply takes into account the final integral effect of charge-sharing depending on signal level. With the charge-sharing PSF results from flat frames, we discuss the effects on photometry in Sec. 4, and finally conclude in Sec. 5.

\section{PTC NONLINEARITY AND AUTOCORRELATION}

We investigate the PTC of AST3 CCD based on the lab flat frames. The PTC is plotted in Fig. 1, and it becomes nonlinear starting from signal $\sim 20,000$ ADU. Therefore in Ref.9 we fitted the data points with signal $<20,000$ ADU with a straight line, whose slope is the inverse of gain. The slope of the best linear fitting in Fig. 1 is 0.61 , corresponding to a gain $=1.64 e^{-} / \mathrm{ADU}$. The PTC tends to deviate from the linear fitting from signal $\sim 20,000$ ADU, and the nonlinearity exceeds $20 \%$ at signal $\sim 60,000$ ADU.

Alternatively a quadratic polynomial has been proved to be an excellent fit to the entire signal range,$\sqrt[4 \mid 6]{6}$ and the coefficient of its linear term can be used to derive the gain. Fig. 1 illustrates that the quadratic function also fits the data of AST3 CCD quite well up to the saturated signal level. The coefficient of its linear term of 0.69 results in gain $=1.44 e^{-} / \mathrm{ADU}$, which is $12 \%$ lower than the previous value from linear fitting to lower signal data. Besides, the nonlinearity occurs obviously even when the signal is lower, and rises to $\sim 30 \%$ at signal $\sim 60,000 \mathrm{ADU}$. 

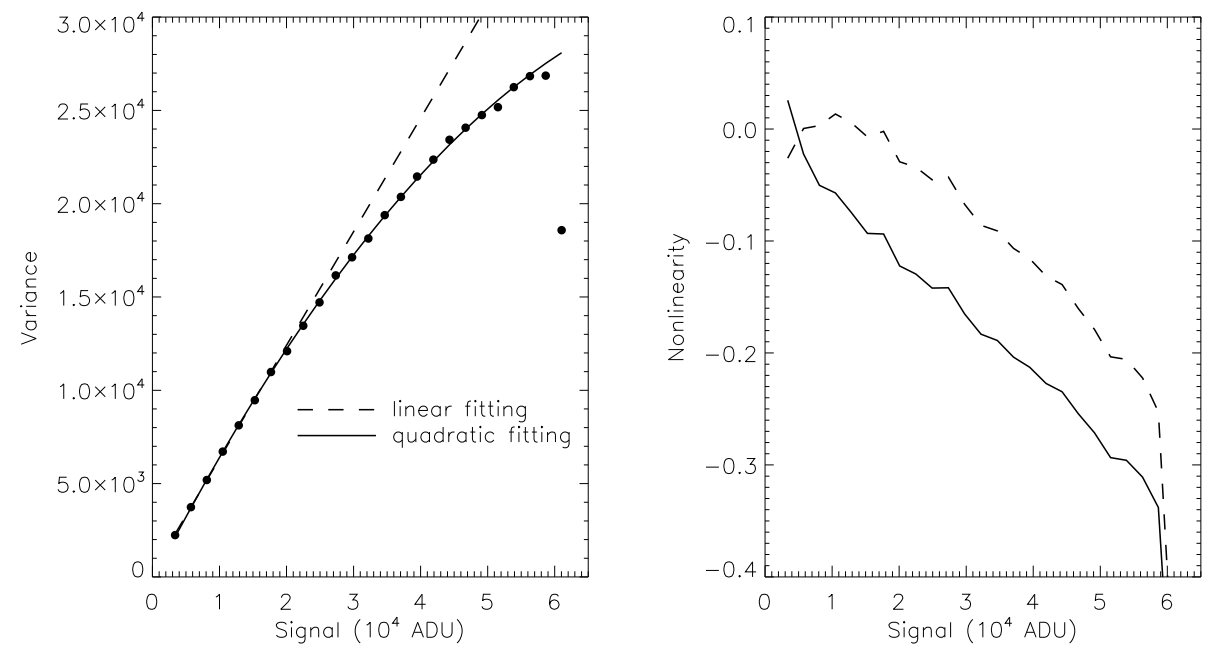

Figure 1. PTC (left) and its nonlinearity (right) of AST3 CCD. In the left panel, the filled circles are data points, and the variance tends to dip as the signal level increases. The sudden drop of variance at the highest signal level is due to saturation. The dashed line is the best linear fitting for signal $<20,000$, and the solid line is the best quadratic fitting to all data points except the saturated one. The right panel shows the normalized residuals with regard to the linear fitting (dashed line) and to only the linear part of the quadratic fitting (solid line).

The sub-Poisson noise strongly suggests that neighboring pixels of flat-fields have statistical correlations. ${ }^{2}$ [4] 7 If $D_{i, j}(i, j$ are the pixel coordinates) is the difference of two uniformly illuminated flat frames, the 2D spatial autocorrelation function $R_{m, n}$, which denotes the correlation between $D_{i, j}$ and $D_{i+m, j+n}$, is calculated by:

$$
R_{m, n}=\frac{\sum_{i=1, j=1}^{i=M-m, j=N-n} D_{i, j} D_{i+m, j+n}}{\sum_{i=1, j=1}^{i=M-m, j=N-n} D_{i, j}^{2}} .
$$

For the ideal Poisson noise, each pixel is independent, thus $R_{m, n}=0$ (except $R_{0,0} \equiv 1$ ). Due to the parity, we only calculate one quadrant of $R_{m, n}$ i.e. $m \geq 0, n \geq 0$, and plot the signal dependent $R_{m, n}$ with only a $4 \times 4$ grid $(0 \leq m \leq 3,0 \leq n \leq 3)$ in Fig. 2. The correlations rise with signal level, decay rapidly with separation, and are larger along Y-axis (CCD columns, vertical transfer) than along X-axis. The nearest vertical neighbors exhibit the strongest correlation, reaching up to about $4.5 \%$ at its highest signal level. The second nearest vertical neighbors and the diagonal neighbors show the second strongest correlation, reaching to about $2 \%$. However, the nearest horizontal neighbors exhibit nearly negligible correlation, whereas the second nearest horizontal neighbors show a merely correlation with a maximum of $\sim 1 \%$. For farther distances, there seems to be weaker or negligible correlations. We believe the positive correlations seen here indicate that the nonlinear PTC is indeed caused by the charge-sharing effect.

\section{CHARGE-SHARING PSF MODEL}

To explain the nonlinearity of PTC and the correlations between pixels, we present a semi-quantitative model on how many charges generated in one pixel are shared with its neighbors. Compared to the model of Ref. 6 , which takes into account the individual electrons and the model of Ref.7, which estimates the pixel boundary displacements perturbed by each neighbor, our model directly derives the final charge-sharing fractions into its neighbors, which is a function of the distance of the neighbors. This function virtually results in an extra PSF, which we call the charge-sharing PSF. Because the Coulomb forces from stored electrons decrease the potential 

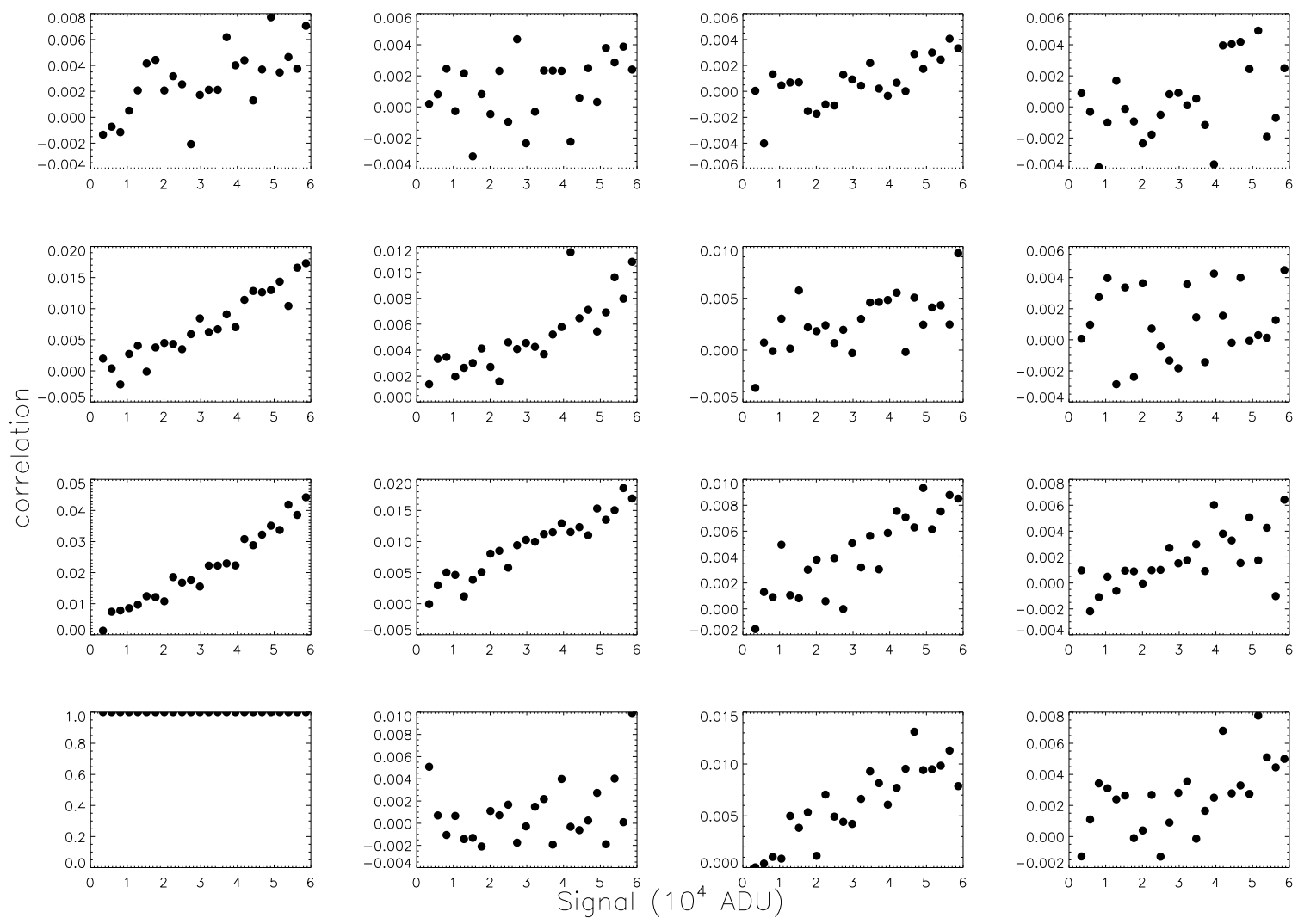

Figure 2. The correlation coefficients $R_{m, n}$ grid for $0 \leq m \leq 3,0 \leq n \leq 3$ as a function of signal level.

wells of pixels, the subsequently generated electrons have some probability to diffuse and may be collected by the neighbor pixels. The shared fraction increases as charges are accumulated, therefore the charge-sharing PSF also depends on the signal level, different from the pure space-varying PSF usually caused by the optics of a telescope and atmospheric seeing.

Deriving the charge-sharing PSF will allow us to quantitatively characterize the CCD's performance as well as to make potential corrections to astronomical images to reduce uncertainties in photometry.

We begin with denoting the charge-sharing PSF as $p_{i, j},-n \leq i, j \leq n$, where $n$ is the size of the PSF, and $p_{i, j} \geq 0, \sum p_{i, j} \equiv 1$. For a certain pixel, its signal without charge sharing is $I_{0,0}$, then after charge sharing when some charges are lost and some extra charges are received, the signal becomes $I_{0,0}^{\prime}$ :

$$
I_{0,0}^{\prime}=\sum_{i=-n, j=-n}^{i=n, j=n} p_{i, j} I_{-i,-j}
$$

Then its variance $V^{\prime}$ for the uniformly illuminated flat frame as a function of raw variance $V$ is:

$$
V^{\prime}=\sum_{i=-n, j=-n}^{i=n, j=n} p_{i, j}^{2} V .
$$

Since $p_{i, j} \geq 0, \sum p_{i, j} \equiv 1$, we have $V^{\prime}<V$ unless $p_{0,0}=1$ which means no charge sharing. So the charge sharing smoothes the image and reduces the variance, as seen in PTC.

The correlation $R_{m, n}$ between $I_{0,0}^{\prime}$ and $I_{m, n}^{\prime}$ can be expressed from charge-sharing PSF by: 


$$
R_{m, n}=\frac{\operatorname{Cov}\left(I_{0,0}^{\prime}, I_{m, n}^{\prime}\right)}{V^{\prime}}=\frac{\operatorname{Cov}\left(\sum_{i, j} p_{i, j} I_{-i,-j}, \sum_{i, j} p_{i, j} I_{m-i, n-j}\right)}{\sum p_{i, j}^{2} V},
$$

where $\operatorname{Cov}$ denotes the covariance, and $\operatorname{Cov}\left(I_{i, j}, I_{m, n}\right)=0$ unless $i=m, j=n$ when $\operatorname{Cov}\left(I_{i, j}, I_{m, n}\right)=V$. Therefore the correlations arise due to the shared charges from the same pixels:

$$
R_{m, n}=\frac{\sum_{i, j} p_{-i,-j} p_{m-i, n-j}}{\sum p_{i, j}^{2}} .
$$

Approximately, we only consider the terms showing significant correlation in Sec. 2 (see Fig. 21), which are $p_{0,0}, p_{0, \pm 1}, p_{0, \pm 2}$, and $p_{ \pm 1, \pm 1}$, respectively. Hence Eq. 5 can be written as:

$$
\begin{aligned}
R_{0,1} & =\frac{2 p_{0,0} p_{0,1}+2 p_{0,1} p_{0,2}}{\sum p_{i, j}^{2}} \\
R_{0,2} & =\frac{2 p_{0,1}^{2}+2 p_{0,0} p_{0,2}}{\sum p_{i, j}^{2}} \\
R_{1,1} & =\frac{2 p_{0,0} p_{1,1}+2 p_{1,1} p_{0,2}}{\sum p_{i, j}^{2}}
\end{aligned}
$$

To solve these PSF coefficients from the PTC and correlation measurements, we take further approximations. Since $p_{0,0}$ is the largest and close to $1, p_{0,0}^{2}$ term dominates the variance in Eq. 3. consequently the remained fraction $p_{0,0}$ can be estimated by:

$$
p_{0,0} \simeq \sqrt{V^{\prime} / V} .
$$

Also allowing for $p_{0,0} \gg p_{0,1} \sim p_{0,2} \sim p_{1,1}$, the sharing fractions to neighbor pixels in Eq. 6 are approximated to:

$$
\begin{aligned}
& p_{0,1} \simeq p_{0,0} R_{0,1} / 2 \\
& p_{0,2} \simeq p_{0,0} R_{0,2} / 2 \\
& p_{1,1} \simeq p_{0,0} R_{1,1} / 2 .
\end{aligned}
$$

It is clear that we can calculate the charge-sharing PSF $p_{i, j}$ from the data we have in Fig. 1 and Fig. 2 ,

However the ideal Poisson variances $V$ estimated by linear fitting to low signal and by the linear term of the quadratic fitting to the entire signal range (Sec 2) vary by a factor of $\sim 10 \%$. We utilize both linear and quadratic fittings to calculate $p_{0,0}$ and subsequent coefficients, and plot them as open squares in Fig. 3.and Fig. 4. respectively. As it has a slightly less nonlinearity, the linear fitting (initial estimates) results in an apparently larger $p_{0,0}$. But both methods end up with shrinking sums of PSF when the signal increases (see Fig. [5), despite that the sums are expected to be invariably unity. Moreover, the sums of PSF from the quadratic fitting suffer greater deviation, whose maximum is near $10 \%$ at the highest signal level, while the maximal deviation from the linear fitting is less than $4 \%$.

To reduce the uncertainties, we employ an iteration method. We re-derive the remained fraction $p_{0,0}$ by subtracting the total loss fraction from 1 :

$$
p_{0,0}=1-2 p_{0,1}-2 p_{0,2}-4 p_{1,1} .
$$

Then we iterate the calculation for the other coefficients according to Eq. 8. Merely three iterations could make the coefficients convergent, and the sum of PSF practically unity (see Fig. 5, and the maximal deviation is less than 0.0001). The convergent PSF coefficients, shown as filled circles in Fig. 3 and Fig. 4, are almost identical 


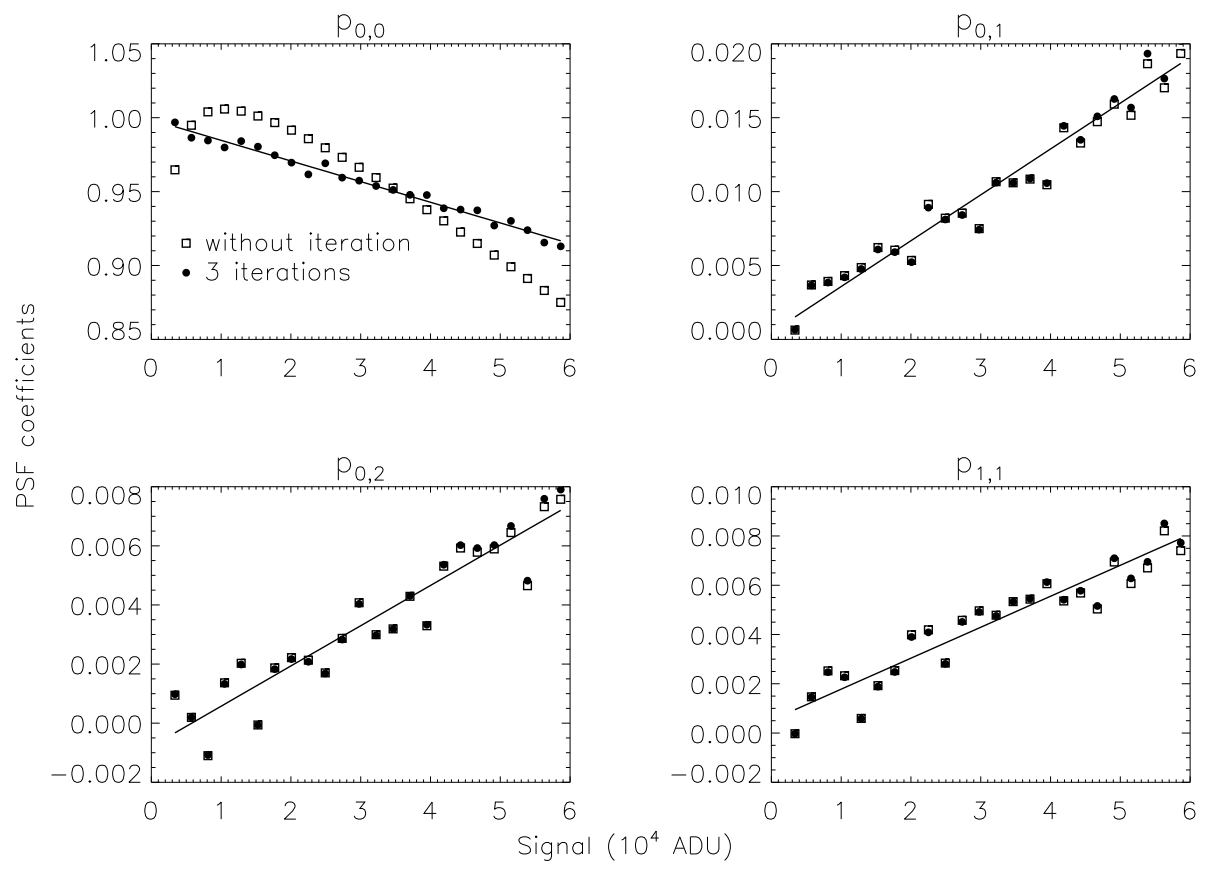

Figure 3. The signal dependent charge-sharing PSF coefficients from the linear fitting (Fig. 1). Open squares are initial estimates and the filled circles are the convergent values after 3 iterations.

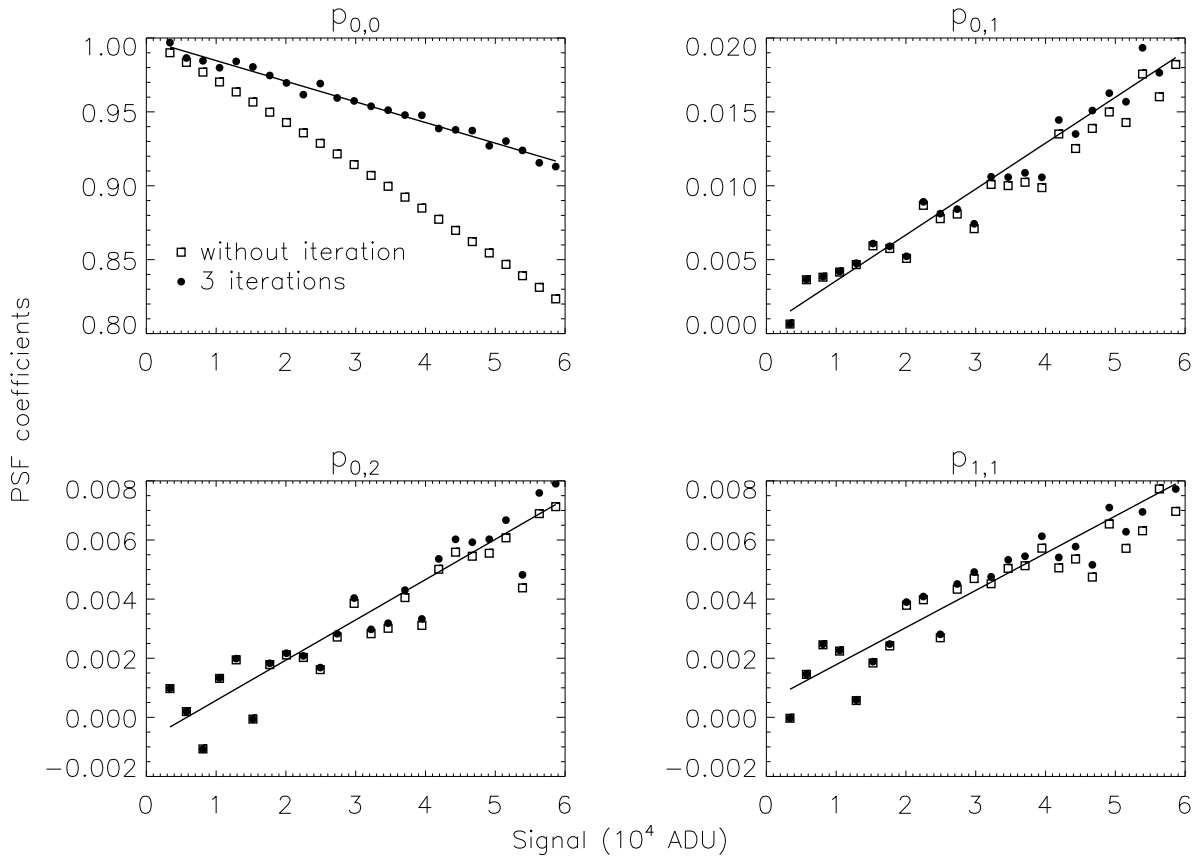

Figure 4. Same as Fig. 3, but for the quadratic fitting to the entire PTC (Fig. 1). 

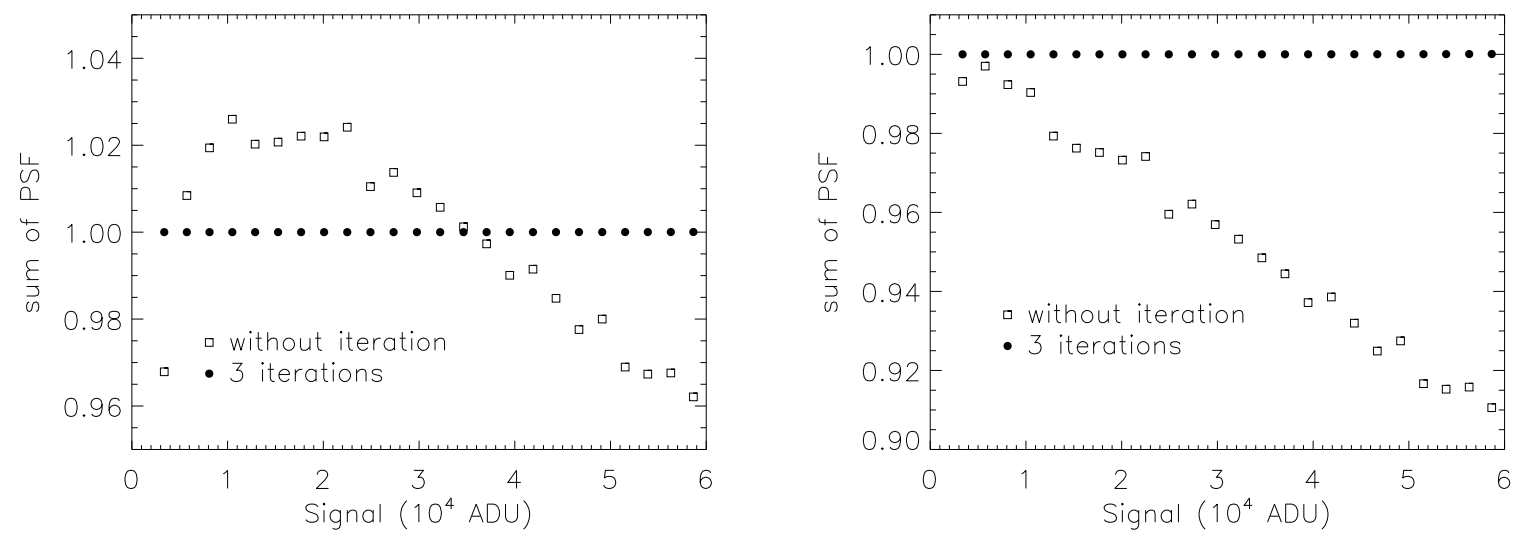

Figure 5. The sum of the charge-sharing PSF coefficients, which is expected to be exact 1. Open squares represent the initial estimations where the ideal Poisson variances $V$ are estimated from PTC (Fig. 1) either by linear fitting (left) or by the linear term of the quadratic fitting (right). The filled circles represent the results after three iterations where the ideal Poisson variances are calculated by the latest derived PSF.

whether the linear or quadratic fitting is used to derive the Poisson variance $V$. In addition, $p_{0,0}$ decreases linearly with the signal level, while the others increase linearly with the signal level. For instance, at the maximal signal level of $6 \times 10^{4} \mathrm{ADU}$, about $9 \%$ electrons in a pixel will diffuse out and be captured by the neighbor pixels: $2 \%$ by each nearest vertical neighbor and $0.8 \%$ by each second nearest vertical neighbor and each diagonal neighbor. These results demonstrate that this charge-sharing PSF model is simple but effective to explain the nonlinear PTC.

On the other hand, the convergent $p_{0,0}$ differs a little from the initial $p_{0,0}$ for the linear fitting (Fig. 3), and the subsequent coefficients do not vary much, either. However, in the case of the quadratic fitting (Fig. (4), the slope of the convergent $p_{0,0}-$ signal relationship becomes less steep than the initial $p_{0,0}$, thus the subsequent coefficients vary a little more for high signal levels. This implies that the quadratic fitting overestimates the Poisson noise, and consequently underestimates the gain. Conversely, the results of $p_{0,0}$ could be used to estimate the Poisson noise and the "gain" parameter more precisely.

\section{EFFECTS ON PHOTOMETRY}

The impact of the charge sharing effects on photometry needs to be investigated. Firstly, the shape of a star will be changed due to this extra PSF. For example, in Fig. 6 the open squares illustrates a star with a Gaussian profile with a Full Width at Half Maximum (FWHM) of 2 pixels and a peak flux of $5 \times 10^{4}$ ADU. Convolved with the charge-sharing PSF derived in Sec. 3, the shape is shown by filled circles in Fig. 6. The resulted shape is more complicate than being just broadened, because the charge-sharing PSF is dependent on signal level. A brighter pixel will lose a larger fraction of electrons, and the peak pixel will lose electrons with the largest fraction. So the final shape is not exactly Gaussian any more, but instead with the peak slightly sliced off. In Fig. 6. the left figure shows how the profile along $\mathrm{Y}$-axis changes. Moreover, the charge sharing effect is much stronger along $\mathrm{Y}$-axis than along $\mathrm{X}$-axis. As a result, the shape tends to be fatter along $\mathrm{Y}$-axis, i.e. the shape is elongated. The ratios of pixel values between raw image and the convolved image (Fig. 6, right) show that the central pixels are abated, while their neighbors along Y-axis are obviously enhanced.

In order to show how the FWHM changes with the peak flux, we simply fit the convolved profiles with a Gaussian profile. As seen in Fig. 7, the FWHM of a star increases linearly with its peak value. For the brightest stars with a peak value of $6 \times 10^{4} \mathrm{ADU}$, their FWHMs are about 0.12 pixel larger than those of the faintest stars, while their shapes are elongated by a factor of more than $3 \%$.

However the FWHM dependence on peak flux varies with the intra-pixel location of a star's centroid. As the centroid shifts inside a pixel, the pixels surrounding the peak pixel will have different signal levels, corresponding 

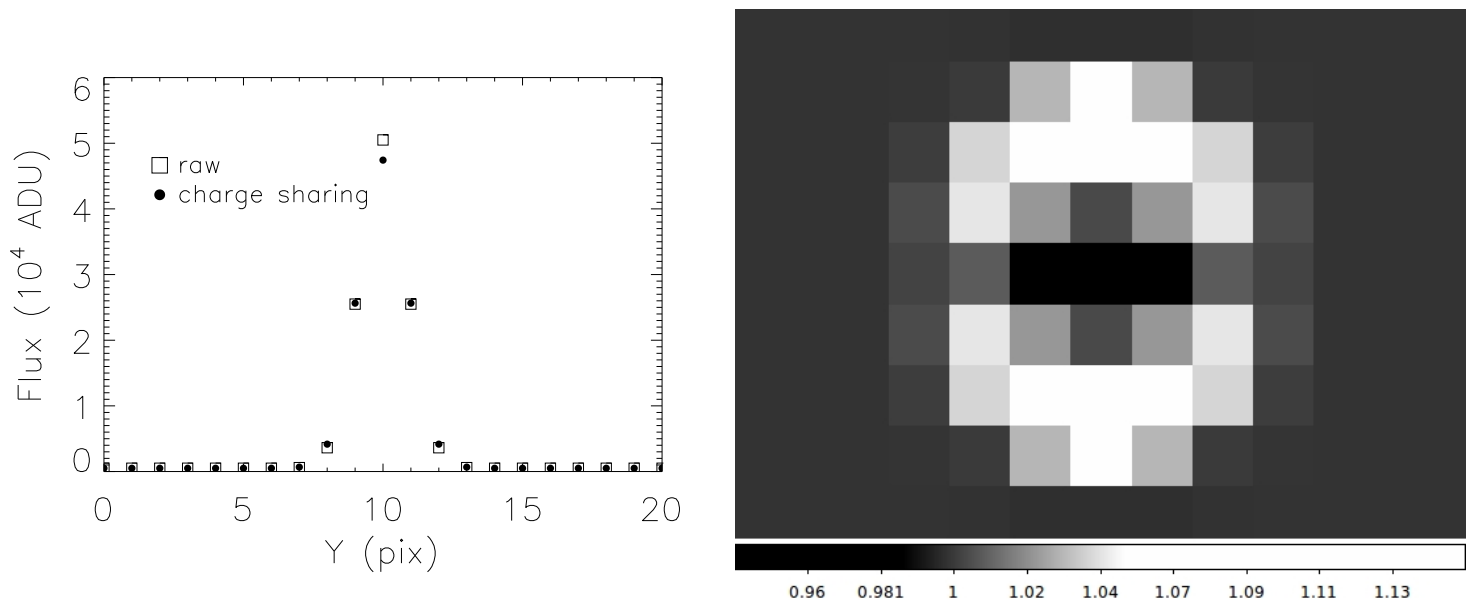

Figure 6. How a Gaussian profile is changed by the charge sharing PSF. Left: the profile along Y-axis, the open squares are the raw profile, and the filled circles are the convolved profile. Right: the ratio of pixel values between raw image and convolved image.
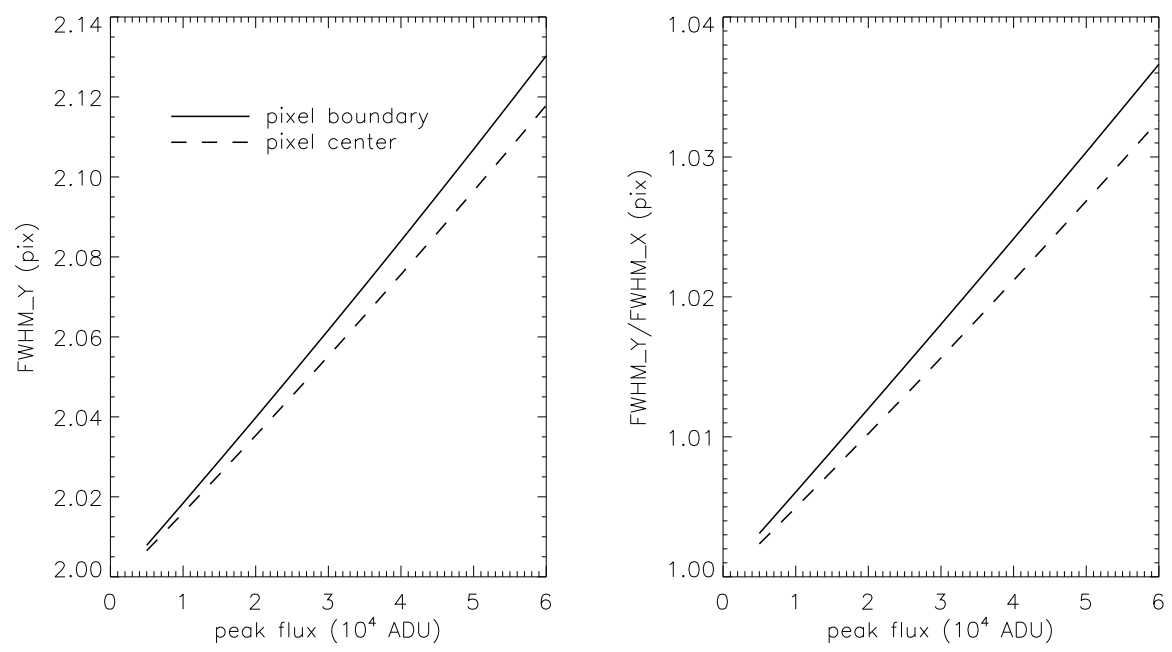

Figure 7. The signal dependent shape: FWHM along Y-axis (left), and the elongation (right). The stars are located at the boundary between two pixels (solid lines), or at the center of a pixel (dashed lines).

to various charge-sharing PSFs. The final profile will consequently vary a little. Fig. 7 illustrates two extremes with simulations: with the star centroid at the pixel boundary and center, respectively. The variations between the two extremes in FWHM and elongation also increase with the peak flux, and their maximums are about 10\%. Although small, this second-order effect may be considered for applications requiring extreme precision on shape measurements.

The flux dependent shape will bias the PSF photometry when the measured star has a different flux from the stars used to derive the PSF. However, the aperture photometry would be less affected, because the charge-sharing distance is essentially short. For bright stars, large apertures are often adopted to improve the signal-to-noise ratio (SNR), in which the charge loss fractions are essentially small. On the other hand, for faint stars, although small apertures are adopted to reduce the noise from background, the intrinsic Poisson noise is still much larger than the bias caused by charge sharing, which is also very weak at low signal levels. One complication would be to carefully define apertures of different sizes for stars of different brightness. The existence of the charge-sharing PSF can affect the photometry using the difference image analysis (DIA, i.e., image subtraction) dramatically, 
if only space-varying PSF is considered. To achieve high precision, the charge-sharing PSF should also be taken into account to derive the convolve kernel. Besides, the flat frames to correct the variations in the pixel-to-pixel sensitivity wil be influenced, because the charge sharing smoothes these variations. It is more critical for dome flat and twilight flat, which are usually obtained at high signal level, while it is insignificant for super-sky flat, whose signal levels are essentially low.

\section{CONCLUSIONS}

We have investigated the PTC of AST3 CCD, and found that it becomes nonlinear at about 1/3 full well. While the linear fitting to PTC only suitable for lower signal range, the quadratic function fits the entire signal range well. However, the slopes of the linear fitting and the linear term of the quadratic fitting vary by about $10 \%$, resulting in gain $=1.64,1.44 e^{-} / \mathrm{ADU}$, respectively. The sub-Poisson noise suggests correlations between neighboring pixels. We derive the $2 \mathrm{D}$ correlation coefficients, and find the correlations increase with the signal level, decay rapidly with the separation (distance), and are larger along Y-axis (CCD columns) than along Xaxis. However, the nearest horizontal neighbors exhibit nearly negligible correlation, whereas the second nearest horizontal neighbors show slight correlation. We do not have an explanation for this phenomena, yet.

The correlation has been proposed to be caused by charge sharing when the Coulomb forces produced by collected charges in a pixel reduce its potential well and repel the subsequent charges. We present a signal dependent charge-sharing PSF model to describe what fractions of charges generated in one pixel are shared with its neighbors. The charge-sharing PSF $p_{i, j}$ denotes the fraction of charges generated in one pixel but finally collected by its neighbor pixel $(i, j)$. According to the correlation results, we approximately take into account $p_{i, j}$ with significant correlations, which are $p_{0, \pm 1}, p_{0, \pm 2}, p_{ \pm 1, \pm 1}$ as well as $p_{0,0}$ which is the remained fraction of charges in the original sharing pixel. Then the nonlinearity of PTC and the correlations can be expressed in terms of $p_{i, j}$. To solve $p_{i, j}$ conveniently, some reasonable approximations are used based on the fact that $p_{0,0} \gg p_{0,1} \sim p_{0,2} \sim p_{1,1}$. Although the linear fitting and the quadratic fitting to the PTC infer different nonlinear level, three iterations successfully make $p_{i, j}$ convergent and consistent. Therefore the convergent $p_{0,0}$ is supposed to derive a more precise gain, which will be investigated in the future.

We demonstrate the shared fraction of charges increases linearly with the signal level, while the remained fraction decreases linearly with the signal level. This signal dependent charge-sharing PSF will alter stars' profiles to be fatter for brighter stars. Besides, the sharing fractions are larger along Y-axis than X-axis, hence the stars' profiles will be elongated. Therefore the PSF photometry will be biased if the measured star has a different flux from the stars used to derive the PSF. It is the same for image subtraction photometry which largely depends on the PSF models. However, we argue that the aperture photometry is less influenced because the charge sharing decay rapidly with the separation distance. In addition, the dome flat and twilight flat are usually taken at high signal levle, therefore will be smoothed by the charge sharing, and should be exposed at appropriate siganl level according to their CCD's charge sharing degree.

Our model is still simple with a relatively small charge-sharing PSF. In the future, we will accurately measure the stars' shape in the images from AST3, by removing other instrumental effects such as the telescope tracking error. Then we could compare our model with other models. Ours can be regarded as the integral effect of the model in Ref.7, nevertheless herein we only consider the signal level of one pixel, without those of its neighbors. In the flat frames, the signal levels in individual pixels are almost the same, and the differences are caused by Poisson noise. In the scientific frames, however, the signal levels vary quite a lot between stars and the sky background. Even for the stars, the signals drop dramatically with the distance from the center. Take all these into account, we will improve our model to be more accurate. In addition, a broader PSF will be considered to include farther neighbors, which also exhibit slight correlations.

\section{ACKNOWLEDGMENTS}

This work has been supported by the National Basic Research Program of China (973 Program) under grand No. 2013CB834900, the Chinese Polar Environment Comprehensive Investigation \& Assessment Programmes under grand No. CHINARE2014-02-03, and the National Natural Science Foundation of China under grant No. 11003027, 11203039, and 11273019. 


\section{REFERENCES}

[1] Janesick, J. R., [Scientific Charge-Coupled Devices], SPIE Press monograph, Washington (2001).

[2] Downing, M., Baade, D., Sinclaire, P., Deiries, S., and Christen, F., "CCD riddle: a) signal vs time: linear; b) signal vs variance: non-linear," Proc. SPIE 6276, 627609 (2006).

[3] Downing, M., B. D. D. S. and Jorden, P., "Bulk silicon CCDs, Point Spread Function and Photon Transfer Curves: CCD testing activities at ESO," Presented at Detectors for Astronomy Workshop (2009).

[4] Downing, M. and Sinclaire, P., "The ccd riddle revisted: Signal versus time linear signal versus variance non-linear," Proc. SDW (2013).

[5] Astier, P., El Hage, P., Guy, J., Hardin, D., Betoule, M., Fabbro, S., Fourmanoit, N., Pain, R., and Regnault, N., "Photometry of supernovae in an image series: methods and application to the SuperNova Legacy Survey (SNLS)," A\&A 557, 55 (2013).

[6] Stefanov, K., "A Statistical Model for Signal-Dependent Charge Sharing in Image Sensors," IEEE Transactions on Electron Devices 61(1), 110-115 (2014).

[7] Antilogus, P., Astier, P., Doherty, P., Guyonnet, A., and Regnault, N., "The brighter-fatter effect and pixel correlations in CCD sensors," Journal of Instrumentation 9, C3048 (2014).

[8] Cui, X., Yuan, X., and Gong, X., "Antarctic Schmidt Telescopes (AST3) for Dome A," Proc. SPIE 7012 , 70122D (2008).

[9] Ma, B., Shang, Z., Wang, L., Boggs, K., Hu, Y., Liu, Q., Song, Q., and Xue, S., "The test of the 10k x 10k CCD for Antarctic Survey Telescopes (AST3)," Proc. SPIE 8446, 84466R (2012). 